\title{
Familiar unbalanced complex rearrangements involving 13 p-arm: description of two cases
}

\author{
Donatella Conconi ${ }^{1}$, Nicoletta Villa ${ }^{2}$, Serena Redaelli ${ }^{1}$, Elena Sala ${ }^{2}$, Francesca Crosti ${ }^{2}$, Silva Maitz ${ }^{3}$, Miriam Rigoldi ${ }^{2}$, \\ Rossella Parini ${ }^{4}$, Leda Dalprà ${ }^{1,2}$, Marialuisa Lavitrano ${ }^{1}$ and Gaia Roversi ${ }^{1,2^{*}}$
}

\begin{abstract}
Background: Copy number variations (CNVs) are largely known today, but their position is rarely established by fluorescence in situ hybridization (FISH) or karyotype analysis.

Case presentation: We described two families with copy number gain in which FISH analysis with the specific subtelomeric probe of chromosome $4 \mathrm{q}$ and $7 \mathrm{q}$ evidenced a third signal at band 13p11.2. Genomic study by array comparative genomic hybridization defined the triple dose segment. In the first case, the duplicate tract is free of known genes, in the second one it contained three expressed genes.

Conclusions: The CNV localization on the short arm of an acrocentric chromosome could explain the lack of phenotypic effect, being known the regulatory role of heterochromatin in the position-effect silencing. Furthermore, we would like to underline the importance of using complementary techniques such as FISH and array-CGH to obtain a better definition of genomic rearrangements.
\end{abstract}

Keywords: Copy number variations, Chromosome 13 p arm, Unbalanced translocation, FISH, Array-CGH

\section{Background}

Copy number variations (CNVs) are well studied and largely known today [1-3]. They may be losses or gains, extremely variable in size, which may or may not affect the phenotypes. For this reason, CNVs could be classified in pathogenetic, benign and with uncertain significance, as defined by online databases (for example http:// dgv.tcag.ca/dgv/app/home, https://decipher.sanger.ac.uk/, or http:/gvarianti.homelinux.net/gvariantib37/index.php).

Nowakowska et al. [4] described how some CNVs considered as de novo, are de facto inherited by parents carrying a cryptic translocation (not detectable with array comparative genomic hybridization technique). In these cases the unbalanced genomic tract, inherited in the affected proband, is not found in its canonical chromosomal position, but is translocated to another chromosome.

\footnotetext{
* Correspondence: gaia.roversi@unimib.it

${ }^{1}$ School of Medicine and Surgery, University of Milano-Bicocca, Monza, Italy

${ }^{2}$ Medical Genetics Laboratory, San Gerardo Hospital, Monza, Italy

Full list of author information is available at the end of the article
}

Instability of the short arm of acrocentric chromosomes $(13,14,15,21,22)$ is well known, in fact qter satellite $Y$ chromosome has considered a normal variant since 1995 [5]. Non-acrocentric chromosomes with satellites instead of telomeres in both $\mathrm{p}$ arm and $\mathrm{q}$ arm have also been described [6]. Furthermore, most of the small supernumerary chromosome markers derives from acrocentric chromosomes [7, 8]. Finally, even Robertsonian translocations, which have an incidence of about 1/1000 newborns, are the results of a rearrangement between acrocentric $\mathrm{p}$ arms [9].

We had the opportunity to study two non-traditional rearrangements that gave rise to partial trisomies without an apparent phenotypic effect. The first case showed a translocation involving chromosomes $4 \mathrm{qter}$ and $13 \mathrm{p}$ and the second involving chromosomes 7qter and 13p. In both cases, conventional cytogenetics analysis showed normal chromosomes, the fluorescence in situ hybridization (FISH) study with specific subtelomeric probes evidenced a third signal on chromosome 13p and finally genomic study by array comparative genomic hybridization $(\mathrm{CGH})$ defined the triple dose segment.

(c) The Author(s). 2018 Open Access This article is distributed under the terms of the Creative Commons Attribution 4.0 International License (http://creativecommons.org/licenses/by/4.0/), which permits unrestricted use, distribution, and 


\section{Cases presentation}

Materials and methods

\section{Cytogenetic and FISH analysis}

Peripheral blood metaphases were obtained from phytohaemagglutinin-stimulated lymphocytes, cultured with Synchro kit (Celbio) according to manufacturer's protocol. Chromosome analysis was carried out applying QFQ banding according to routine procedures, and karyotypes were reconstructed following the guidelines of ISCN 2016 [10].

FISH analysis was carried out according to the manufacturer's protocol for specific subtelomeric probes (Kit Chromoprobe Multiprobe-T System, Cytocell).

\section{Array-CGH}

Genomic copy number analysis was performed with array-CGH using the SurePrint G3 Human Genome $\mathrm{CGH}+$ SNP Micro-array Kit, $4 \times 180 \mathrm{~K}$ (Agilent Technologies) following the manufacturer's recommendations. The target DNA was extracted from the peripheral blood by Wizard Genomic DNA purification kit (Promega Corporation). DNA control reference was provided by Agilent (Agilent Technologies). The arrays were scanned at 3- $\mu \mathrm{m}$ resolution using an Agilent microarray scanner and analyzed using CyoGenomics 3.0 software (Agilent Technologies). The aberration detection method 2 (ADM-2) algorithm was used to compute and assist in the identification of aberrations for a given sample.

Significant chromosomal aberrations were determined using the algorithm ADM-2 with a threshold of 5 and a minimum absolute average $\log 2$ ratio of 0.25 . Putative chromosome copy number changes were defined by intervals of 3 or more adjacent probes and were considered as being duplicated or deleted when results exceeded 0.25. All nucleotide positions were based on the Human Reference Sequence Assembly, February 2009 GRCh37/hg19 of the UCSC Genome Browser (http://genome.ucsc.edu/).

\section{Cases description \\ Case 1}

The proband was the first child of a non consanguineous couple. He was born at $31+6$ weeks of gestation by caesarean section due to maternal preeclampsia. At birth, a respiratory distress occurred and a feeding by gavage for three weeks was introduced. He showed growth difficulties and psychomotor delay. At 17 months prominent subcutaneous nodules next to hand joints, ankles, knees, spine were present, associated with joint swelling, neurological deterioration, cachexia due to feeding problems and mitralic regurgitation. The baby died at 23 months of age.

Farber disease was hypothesized and successively confirmed by undetectable levels of acid ceramidase
(Laboratory prof. Thierry Levade, Toulose). Molecular analysis of $\mathrm{N}$-acylsphingosine amidohydrolase (ASAH1) gene (8p22) showed the splicing mutation c. $648+1 \mathrm{G}>\mathrm{C}$ in intron 8 and the missense mutation c.1085C $>\mathrm{G}$ (p.Pro362Arg) in exon 13. The phenotype was consistent with the molecular findings. Parents were both carriers for this autosomal recessive disorder.

At 12 months old, conventional karyotype analysis on peripheral blood evidenced an apparently normal karyotype The analysis of subtelomeric regions by means of FISH revealed the presence of a partial trisomy of the terminal region of the chromosome $4 \mathrm{q}$ located at $13 \mathrm{p}$, resulting in a derivative chromosome 13 . The ISCN description was: ish $\operatorname{der}(13) \mathrm{t}(4 ; 13)(\mathrm{q} 35 ; \mathrm{p} 11.2)(\mathrm{q} 35+)$ (DJ9 $63 \mathrm{k} 6+$ ) (Fig. 1a, b). The father and the paternal grandmother were carriers of the same unbalanced translocation (Fig. 1c), as determined by FISH analyses (data not shown).

In order to understand if $4 \mathrm{q}$ trisomy could be considered a polymorphism, an array-CGH analysis was performed in the father (Fig. 1d). Array-CGH analysis confirmed the gain at $4 \mathrm{q}$, consisting in 4 probes partially overlapped the FISH probe. The trisomic region, of about $78 \mathrm{~kb}$, is included within the FRG1-divergent transcript, a long non coding RNA (Fig. 2a, b). The $180 \mathrm{~K}$ array slide covers an additional region of other 6 disomic probes (about $142 \mathrm{~kb}$ ), the last of which maps about $195 \mathrm{~kb}$ apart from the 4qter (region uncovered by arrayCGH probes).

\section{Case 2}

This proband was investigated as the father of a single child affected by psychomotor delay and moderate facial dismorphisms, in whom subtelomeric FISH analysis identified a de novo loss of 9q.

The same investigation in the proband showed an additional signal for the specific subtelomeric $7 \mathrm{q}$ probe on chromosome 13p, giving rise to a derivative chromosome 13 with a segmental $7 q$ trisomy. The ISCN description was: ish $\operatorname{der}(13) \mathrm{t}(7 ; 13)(\mathrm{q} 36 ; \mathrm{p} 11.2)(\mathrm{q} 36+)(2000$ a5+) (Fig. 3a, b). FISH analyses allowed us to define the maternal inheritance of the rearrangement (data not shown), that was not transmitted to the affected child (Fig. 3c). The familial history was uneventful, neither consanguinity nor genetic diseases were reported.

In order to better characterize a trisomic segment, array-CGH analysis was performed in the proband, confirming a duplication of about $525 \mathrm{~Kb}$ at $7 \mathrm{q} 36.3$ (from nt $158,599,150$ to nt $159,124,131$ ) (Fig. 3d). This region is covered by 54 oligo and 7 SNPs probes with a copy number of 3 and includes the 7q telomeric specific FISH probe (from nt $158,859,151$ to nt $158,958,164$ ) (Fig. 4a). The exon 1 of ESYT2 (OMIM616691) and the whole 

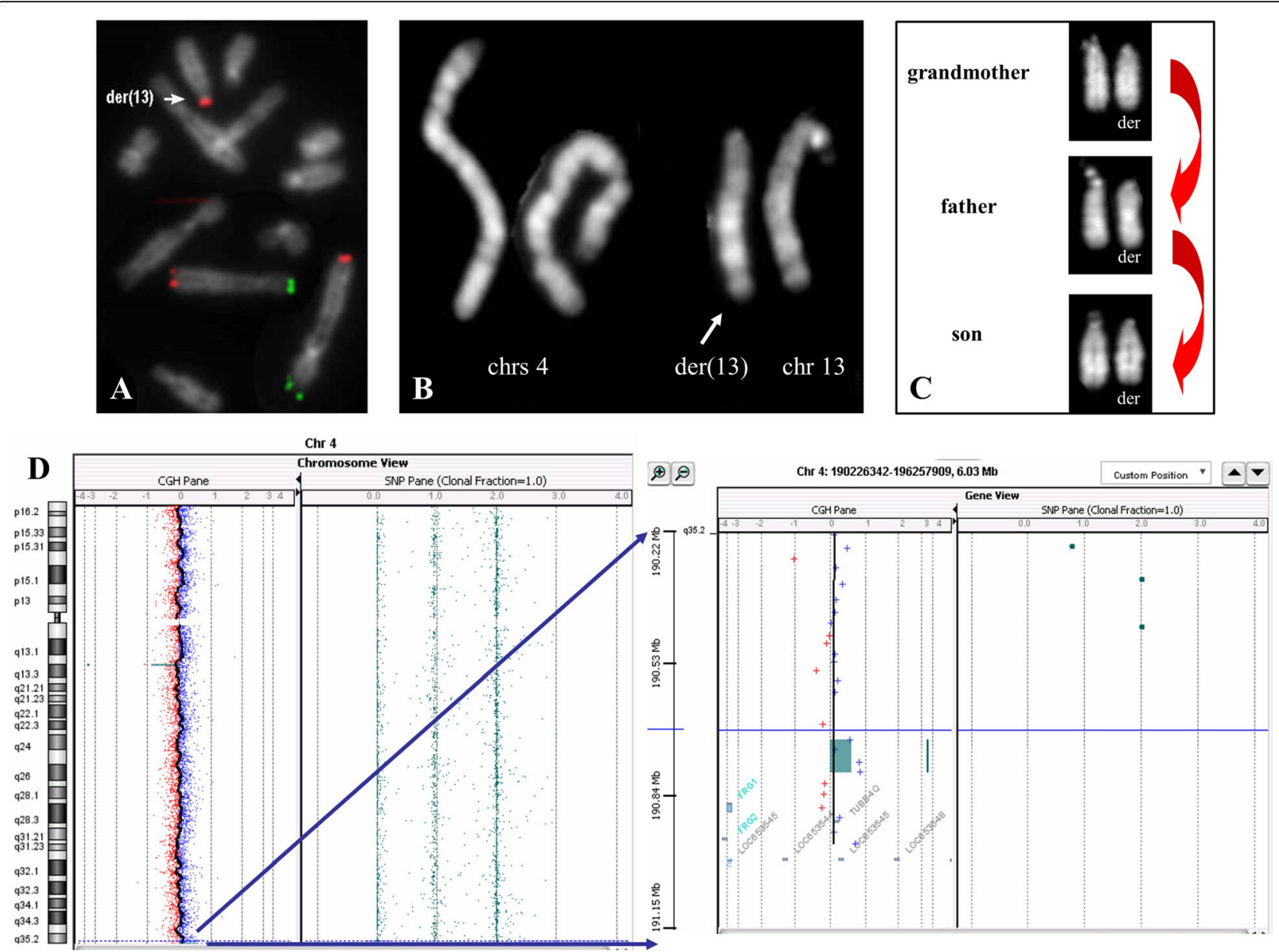

Fig. 1 Genetic characterization of the first case. a FISH analysis with chromosome 4 specific subtelomeric probes: 4pter (green signals) and 4qter (red signals). b Proband's QFQ-banded chromosomes 4 and 13 (700-band levels). c Normal (left) and derivative (right) chromosomes 13 in the proband, his father and grandmother. $\mathbf{d}$ Array-CGH view of chromosome 4 and enlargement of the duplicated region

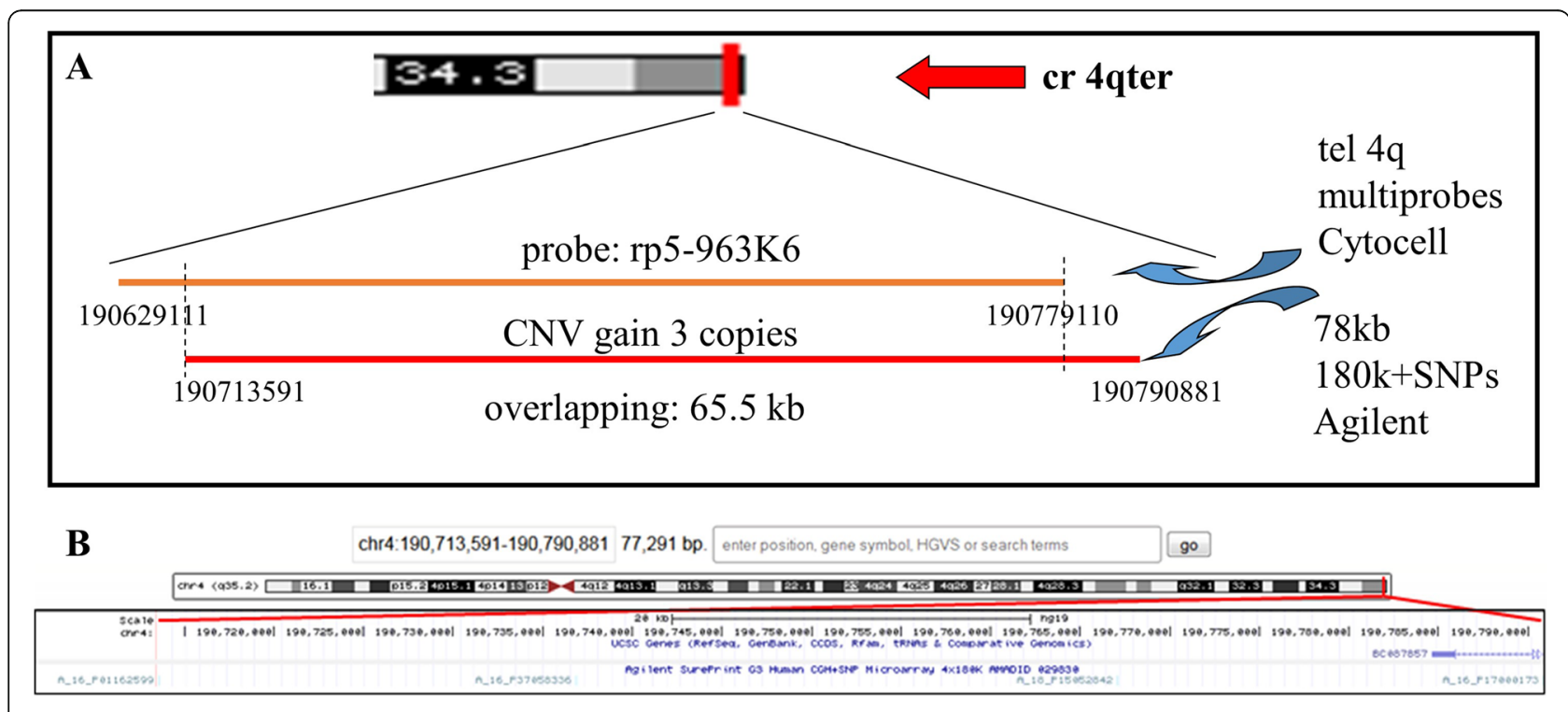

Fig. 2 First CNV description. a 4q subtelomeric FISH probe sequence and CNV position. b UCSC region of the trisomic segment 

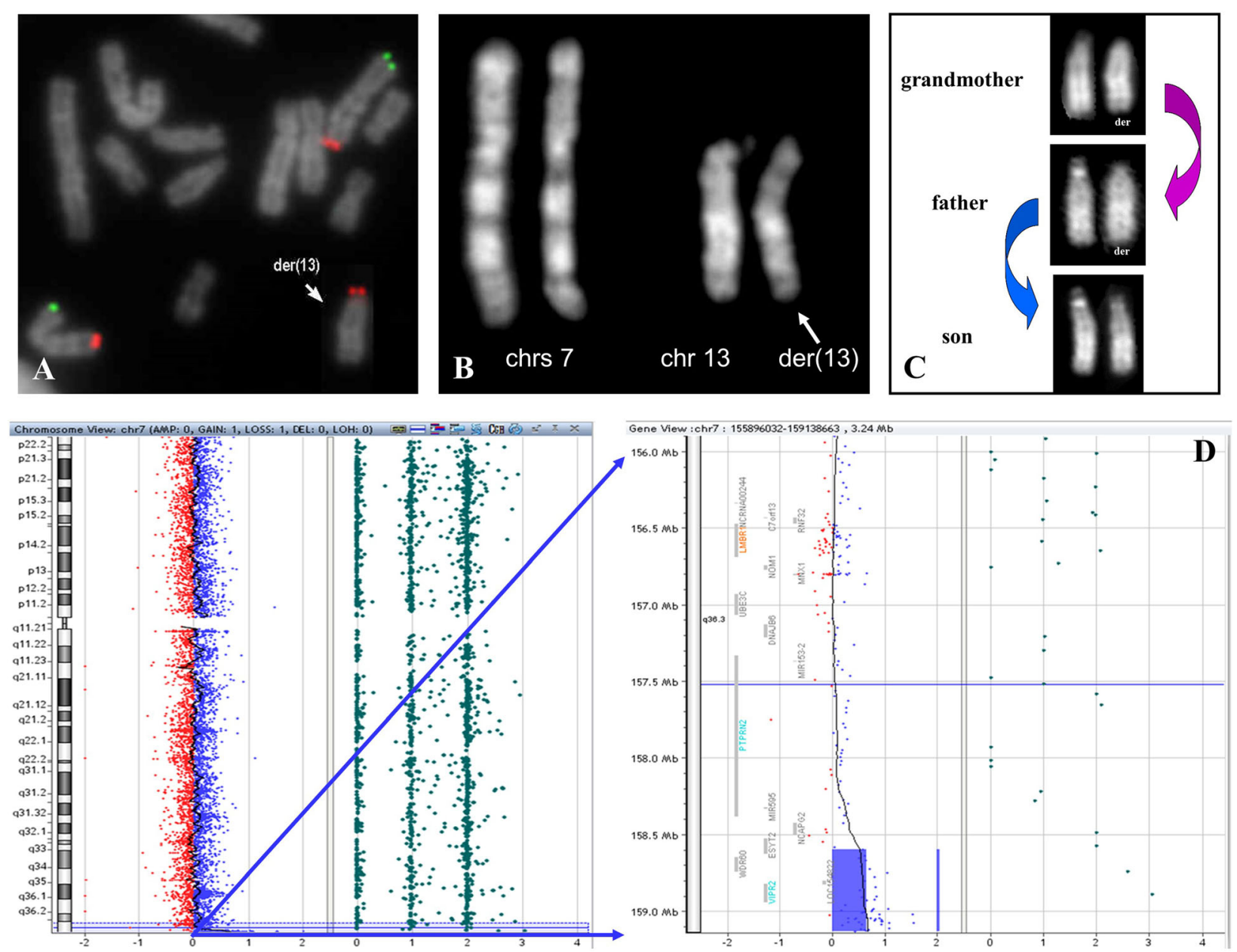

Fig. 3 Genetic characterization of the second case. a FISH analysis with chromosome 7 specific subtelomeric probes: 7 pter (green signals) and 7qter (red signals). b Father's QFQ-banded chromosomes 7 and 13 (550-band levels). c Chromosomes 13 transmission in the child, his father and his grandmother. D) Array-CGH view of chromosome 7 and enlargement of the duplicated region

WDR60 (OMIM615462) and VIPR2 (OMIM601970) genes map within the region (Fig. 4b).

\section{Discussion and conclusions}

Several studies about CNVs have been reported, but in very few cases chromosomes were performed in order to verify their position. Here we described two families with a copy number variation in which the gained segment is translocated to the 13p11.2 region that loses satellite sequences. This type of translocation could be underestimated because cytogenetically unrecognizable, in contrast to the cases in which a derivative chromosome acquires satellite sequences at the end of short or long arm (ps or qs chromosome).

The recurrence of cases with rearrangements involving acrocentric $\mathrm{p}$ arms and terminal region of other chromosomes suggests a predisposing mechanism. Intriguingly, Cazaux et al. [11] reported that the proximity between telomeres, centromeres and rDNA clusters of acrocentric chromosomes during meioses might facilitate their sequence homogenization by non-homologous recombination, according to the model of chromosome pairing proposed by Scherthan et al. [12].

In the first case the duplicate segment does not contain known genes to date, but is included within the FRG1-divergent transcript, a long non coding RNA. This $\mathrm{CNV}$ is reported, with higher size, in Troina Database and in the Database of Genomic Variants (DGV) as benign.

In the second case, the $\mathrm{CNV}$ is indicated as polymorphic variant in $0.04 \%$ of cases of DGV gold standard variant; Troina Database shows four overlapping CNVs all classified as benign and finally Decipher reports four CNVs, one inherited with unknown classification, one benign inherited, one of uncertain significance and the last inherited with autistic phenotype (partial uncertain significance). The trisomic segment contains three expressed genes: WDR60, associated to short-rib thoracic dysplasia 8 


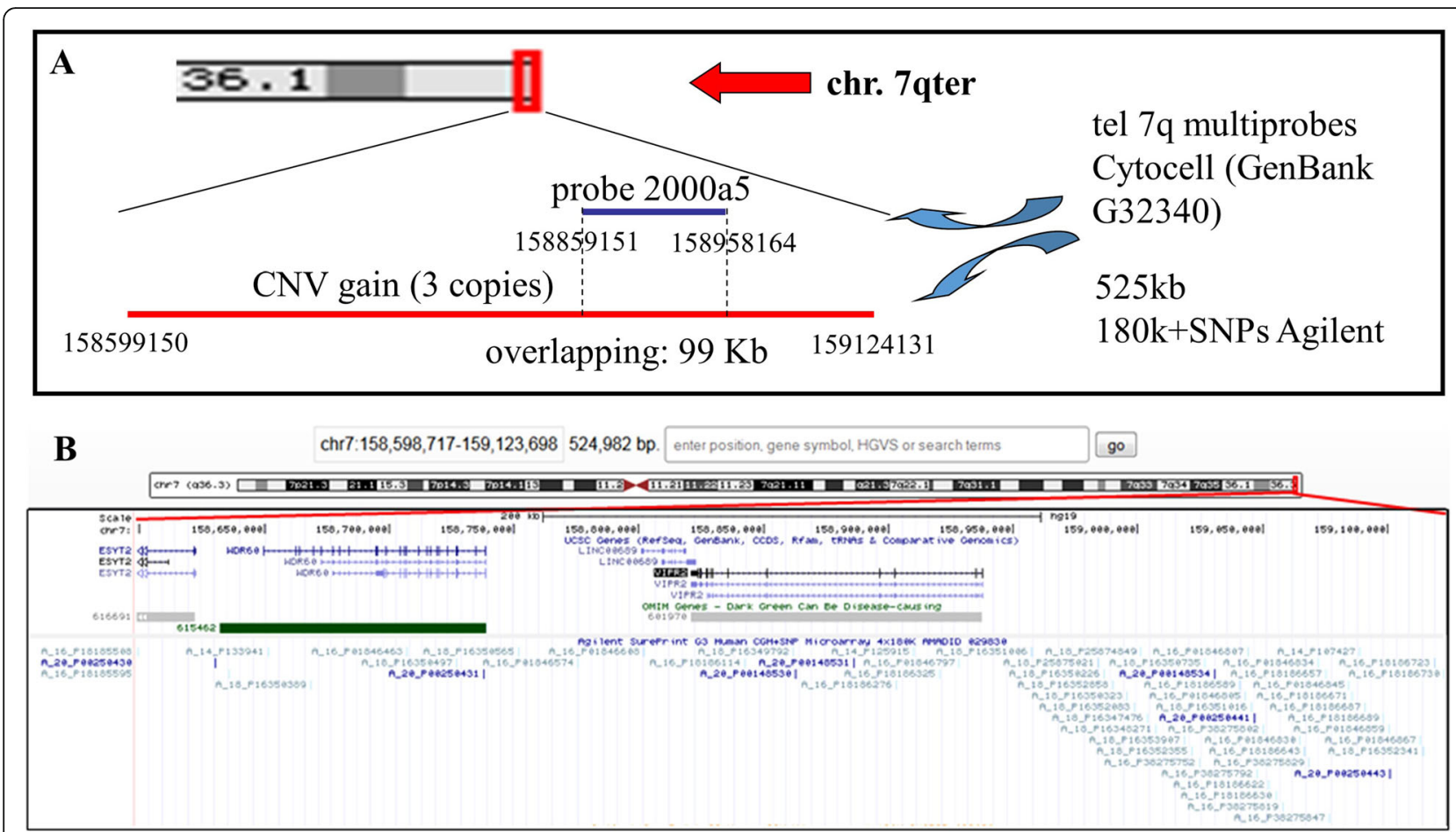

Fig. 4 Second CNV description. a 7q subtelomeric FISH probe sequence and CNV position. b UCSC and OMIM genes in the trisomic region

with or without polydactyly, ESYT2, that encodes for extended synaptotagmin-like protein 2 and is not associated to pathology and VIPR2, that has been reported in association with schizophrenia [13].

The copy number gain localization on the short arm of an acrocentric chromosome in this case could explain the lack of phenotypic effect, being known the regulatory role of heterochromatin in the position-effect silencing.

We would like to stress the importance of using complementary techniques such as FISH and array-CGH to obtain a better definition of genomic rearrangements.

\section{Abbreviations}

CGH: Comparative Genomic Hybridization; CNV: Copy Number Variation; DGV: Database of genomic variants; FISH: Fluorescence In Situ Hybridization

\section{Availability of data and materials}

The datasets used and/or analyzed during the current study available from the corresponding author on reasonable request.

\section{Authors' contributions}

DC contributed to design the study, collected the literature data and wrote a part of the manuscript. NV performed genetic studies on the patients, contributed to design the study, collected the literature data and wrote a part of the manuscript. SR, FC, ES performed clinical evaluation of the patients. SM, MR, RP performed genetic studies on the patients. LD, ML, GR contributed to design the study, wrote a part of the article and critically read the manuscript. All authors read and approved the final manuscript.

Ethics approval and consent to participate Not applicable.

\section{Consent for publication}

Written informed consent was obtained from the patient's parents for publication of this case report and any accompanying images.

\section{Competing interests}

The authors declare that they have no competing interests.

\section{Publisher's Note}

Springer Nature remains neutral with regard to jurisdictional claims in published maps and institutional affiliations.

\section{Author details}

${ }^{1}$ School of Medicine and Surgery, University of Milano-Bicocca, Monza, Italy. ${ }^{2}$ Medical Genetics Laboratory, San Gerardo Hospital, Monza, Italy. ${ }^{3}$ Pediatric Genetic Unit, Pediatric Department of Monza Brianza per il Bambino e la sua Mamma (MBBM) Foundation, San Gerardo Hospital, Monza, Italy. ${ }^{4}$ Pediatric Department of Monza Brianza per il Bambino e la sua Mamma (MBBM) Foundation, San Gerardo Hospital, Monza, Italy.

Received: 4 June 2018 Accepted: 29 August 2018

Published online: 06 September 2018

\section{References}

1. Redon R, Ishikawa S, Fitch KR, Feuk L, Perry GH, Andrews TD, et al. Global variation in copy number in the human genome. Nature. 2006;444(7118): 444-54.

2. Conrad DF, Pinto D, Redon R, Feuk L, Gokcumen O, Zhang Y, et al. Origins and functional impact of copy number variation in the human genome. Nature. 2010;464(7289):704-12.

3. Zarrei M, MacDonald JR, Merico D, Scherer SW. A copy number variation map of the human genome. Nat Rev Genet. 2015;16(3):172-83.

4. Nowakowska BA, de Leeuw N, Ruivenkamp CA, Sikkema-Raddatz B, Crolla $J A$, Thoelen $R$, et al. Parental insertional balanced translocations are an important cause of apparently de novo CNVs in patients with developmental anomalies. Eur J Hum Genet 2012;20(2):166-170. 
5. Mitelman F and International Standing Committee on Human Cytogenetic Nomenclature (1995) ISCN 1995: an international system for human cytogenetic nomenclature (1995): recommendations of the International Standing Committee on Human Cytogenetic Nomenclature, Memphis, Tennessee, USA, October 9-13, 1994. Karger.

6. Sarri C, Douzgou S, Gyftodimou Y, Tümer Z, Ravn K, Pasparaki A, et al. Complex distal 10q rearrangement in a girl with mild intellectual disability: follow up of the patient and review of the literature of non-acrocentric satellited chromosomes. Am J Med Genet A. 2011;155A(11):2841-54.

7. Dalprà L, Giardino D, Finelli P, Corti C, Valtorta C, Guerneri S, et al. Cytogenetic and molecular evaluation of 241 small supernumerary marker chromosomes: cooperative study of 19 Italian laboratories. Genet Med. 2005;7(9):620-5.

8. Liehr T, Mrasek K, Dufke A, Rodríguez L, Martínez Guardia N, et al. Small supernumerary marker chromosomes--progress towards a genotypephenotype correlation. Cytogenet Genome Res. 2006;112(1-2):23-34.

9. Jarmuz-Szymczak M, Janiszewska J, Szyfter K, Shaffer LG. Narrowing the localization of the region breakpoint in most frequent Robertsonian translocations. Chromosom Res. 2014;22:517-32.

10. McGowan-Jordan J, Simons A, Schmid M, International standing committee on human Cytogenomic nomenclature. ISCN: an international system for human cytogenomic nomenclature. Basel; New York: Karger; 2016.

11. Cazaux B, Catalan J, Veyrunes F, Douzery EJ, Britton-Davidian J. Are ribosomal DNA clusters rearrangement hotspots?: a case study in the genus Mus (Rodentia, Muridae). BMC Evol Biol. 2011;11:124.

12. Scherthan H, Weich S, Schwegler H, Heyting C, Härle M, Cremer T. Centromere and telomere movements during early meiotic prophase of mouse and man are associated with the onset of chromosome pairing. J Cell Biol. 1996;134(5):1109-25.

13. Vacic V, McCarthy S, Malhotra D, Murray F, Chou HH, Peoples A, et al. Duplications of the neuropeptide receptor gene VIPR2 confer significant risk for schizophrenia. Nature. 2011:471(7339):499-503.

Ready to submit your research? Choose BMC and benefit from:

- fast, convenient online submission

- thorough peer review by experienced researchers in your field

- rapid publication on acceptance

- support for research data, including large and complex data types

- gold Open Access which fosters wider collaboration and increased citations

- maximum visibility for your research: over $100 \mathrm{M}$ website views per year

At $\mathrm{BMC}$, research is always in progress.

Learn more biomedcentral.com/submissions 